\title{
THE AFFERENT COURSE OF THE VAGUS NERVE IN THE MEDULLA OBLONGATA
}

\author{
Miyoshi Urabe, M.D., Shin Yamazaki, M.D. and Kimpei Araki, M.D. \\ Dept. of Surg., School of Med. \\ Univ. of Kanazawa
}

\section{INTRODUCTION}

The present authors have previously reported the intramedullary course of afferent fibres of the glossopharyngeal and vagus nerves, according to the results of the study of serial sections in Marchi's staining of the medulla, where the degeneration was caused by cutting the glossopharyngeal and vagus nerves. The newly-discovered course of the degenerated afferent fibres of the glossopharyngeal and vagus nerves was emphasized which had not been noticed before by any authors. At the level where the sensory root of the glossopharyngeal and vagus nerves entered the medulla, fascicles of medium-sized or thick degenerated fibres were recognized to pass through the solitary tract dorsalward into the most dorsal part of the basal grey substance along the medial border of the vestibulospinal tract. The degeneration of these newly-discovered fibres occurred when the glossopharyngeal and vagus nerves were cut between the ganglia as well as at the intracranial roots.

The triangular nucleus has long been regarded as an autonomic centre in the medulla, and was named "Vestibulär-alimentäres Reflexzentrum" by Spitzer, who thought the centre regulated the mechanism of taking nutrition into the body. It was also well known that both the glossopharyngeal and vagus nerves had close relation to digestion. Therefore, it was significant that the newly-discovered course originated in the nodose or petrous ganglion which regulated the visceral afferent components, and that the course had contact not only with the solitary tract and solitary nuclei, but also with the area of the triangular nucleus.

In the present report, the authors are going to describe the termination of the new fibres, and discuss the relationship between the new fibres and the vestibular nerve, as well as the relationship between the new fibres and the afferent fibres from the spinal cord. 


\section{Material and Methods}

Eight adult mongrel dogs were used. All the animals were operated upon under Buthalitone sodium anesthesia $(30-40 \mathrm{mg} / \mathrm{kg})$. The cutting was done upon the intracranial roots and the interganglia of the vagus nerve; the root of the acoustic nerve; the posterior root, a quarter of the anterior area, and the anterolateral funiculi, of the spinal cord. The animals were sacrificed 8-18 days after the operation. The spinal cord, the medulla, the pons, and the midbrain were taken out, and were fixed in Müller's solution for 4-5 days. Then they were stained by Marchi's principle and were observed in serial sections $(50 \mu)$. Great care was taken to avoid trauma to the tissues at the time of the operation and the autopsy.

The afferent course of the fibres could be traced by observing the degeneration developed after the infliction of the damage.

\section{OBSERVATIONS}

The pathway of the newly-found fibres, which originated in the nodose ganglion of the vagus nerve was accurately perceived on the cross-sections of the medulla in the cases of No. 19,20, and 21, and on the sagittal sections of the medulla in the case of No. 22. It was clarified in the cases of No. 17 and 18 that the distribution of the newly-found course was confined within the area where the degeneration appeared after cutting the root of the vestibular nerve. The relationship between the newly-found course of the vagus nerve and the afferent fibres from the spinal cord was observed in the cases of No. 13, 15, 19, 20, and 21. The afferent fibres from the spinal cord were not intermingled with the new fibres. However, in the rostral part of the nucleus fasciculi gracilis and in the caudal part of the triangular nucleus, there was a clustering of degenerated granules caused by cutting the fibres from the lower spinal cord. These areas of the degenerated granules were situated in the medulla very close to the newlyfound course of the vagus nerve.

Dog 22. The left vagus nerve was cut between the jugular and the nodose ganglia.

The tissue from the lower part of the pons up to the upper part of the spinal cord was taken as a specimen. The serial sections were made from the out-side on the sagittal plane.

Looking at the serial sections from the lateral to the medial ones, in the first few sections several small groups of the degenerated granules appeared in the shape of branchia at the rostral part of the medulla where the root of the vagus nerve entered the medulla. As the section came medialward, the degenerated fibres ran medialward or dorsomedialward in the medulla keeping almost the same plane. They rounded the dorsal part of the trigeminospinal tract, some of 
them passing through the dorsal part of it and entering the solitary tract. As the section proceeded medialward, the small fascicles of degenerated fibres were found to pass through the ventral part of the trigeminospinal tract, also. The section proceeding further medialward, the lateral nucleus of the solitary tract began to appear on the rostral side, containing degenerated granules, and the degenerated fibres which descended into the solitary tract were also recognized.

The sagittal plane does not run completely parallel with the long axis of the solitary tract, that is, the solitary tract makes a gentle convex toward the medial side. Therefore, as the sagittal sections go from lateral to medial, the solitary tract reveals its rostral- and caudal-ends in the first few sections; later it reveals its intermediate part. The solitary tract forms a fascicle in the shape of a band which runs rostrocaudally in the medulla, starting a little below the lower end of the pons. It is adjacent to the vestibulospinal tract and the basal grey substance on the posterior side, and also close to the solitary nucleus and the reticular formation on the ventral side.

Now, a fascicle of the degenerated fibres which ran almost horizontally dorsomedialward from the lateral side as described above, entered the solitary tract at its rostral part, and then turned at a right angle down through the solitary tract. In the lower part of the medulla, the end of the degenerated fibres in the solitary tract was separated into two fascicles at the lateral border of the ala cinerea. As the section came toward the more medial side, the degenerated fibres in the ventral area to the rootlet of the vagus nerve moved dorsocaudally into the solitary tract. In these sections, several degenerated fibres were seen

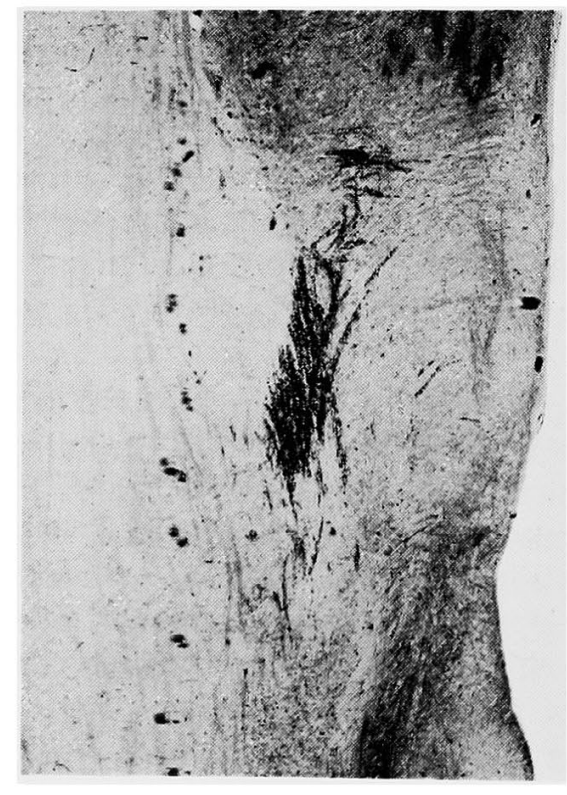

Fig. 1. Dog 22. (89)* Some fascicles of the degenerated fibres run dorsalward from the rostral part of the solitary tract to the basal grey substance. This section is adjacent laterally to the section of Fig. 4.

* The number in parentheses shows a sagittal section of the medulla. Section numbers start from the lateral side and proceed to the medial side in the case of Dog 22. 
to run parallel with the solitary tract, at the level of the rostral part of the tract, near the dorsal border of the medulla.

In the sections of the more medial side where the basal grey substance, as well as the medial side of the rostral part of the solitary tract, began to appear. the degenerated fibres were observed spreading from the rostral part of the solitary tract to the dorsally located basal grey substance in several directions, rostral-, horizontal- and caudal-ward. Some of them made a parabola, while others made a straight line (Fig. 1). As the section went on, this phenomenon became much more distinct. Some degenerated fibres ran into the area where the basal grey substance touched the bottom of the fourth ventricle. Some fibres reached the basal grey substance, making several parabola figures. Some fibres, after starting from the rostral part of the solitary tract, passed dorsally, making a curve, and entered the lower solitary tract again. Some fibres seemed to go round the small luminous nucleus located in the dorsal area to the solitary tract (Fig. 2, 3, 4).

As the section came more medialward, the solitary tract became invisible in its rostral part. The medial nucleus of the solitary tract looked luminous and a little caudalward from this nucleus, the thick fascicle of the degenerated fibres was seen which went down through the solitary tract. In the sections of this plane, there were no degenerated fibres running dorsalward from the solitary tract. A little caudalward the lateral nucleus of the solitary tract which contained the large-sized cells was secn at the level of the rostral edge of the inferior olivary nucleus. The fine granules were gathered conspicuously in this nucleus. Some of

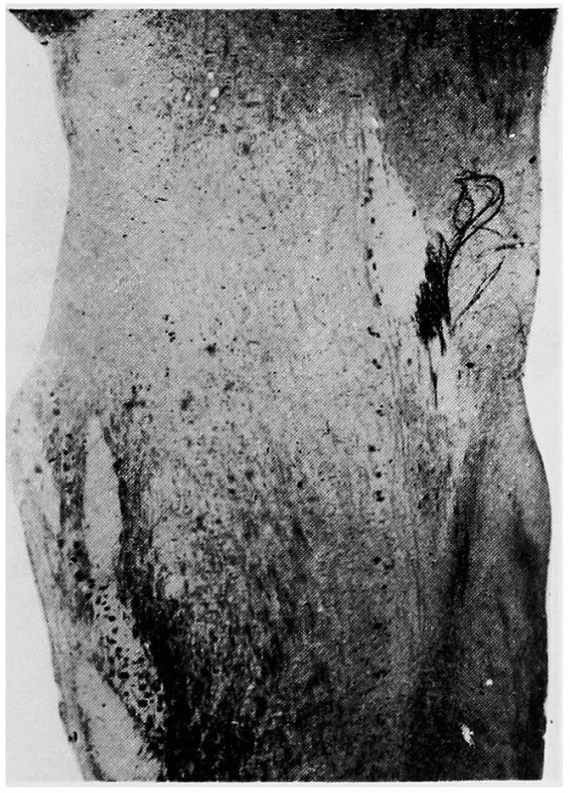

Fig. 2. Dog 22. (90) A number of the degenerated fibres run to the basal grey substance from the rostral part of the solitary tract. 


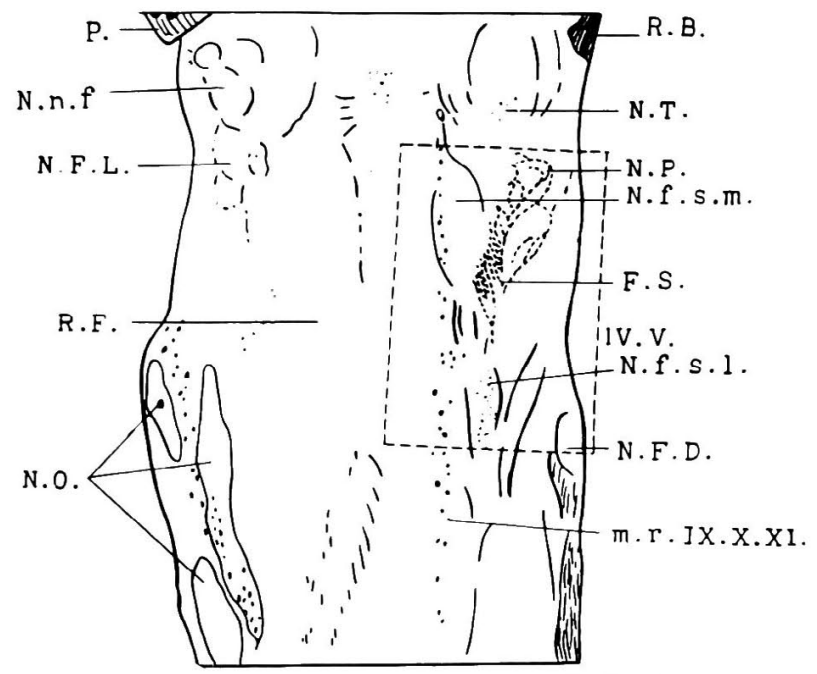

Fig. 3. Schematic illustration of Fig. 2.

R. B.: Restiform body.

N. T.: Triangular nucleus.

N. f. s.m .: Medial nucleus of the solitary tract.

IV. V.: The fourth ventricle.

N.f. s. 1.: Lateral nucleus of the solitary tract.

N. F. D.: Nucleus of the dorsal funiculus. m. r. IX.X. XI. X.: Motor roots of the glossopharyngeal, vagal, and accessory nerves.

P.: Pons.

N. n. f.: Nucleus of the facial nerve.

N. F. L.: Nucleus of the lateral funiculus.

R. F.: Reticular formation.

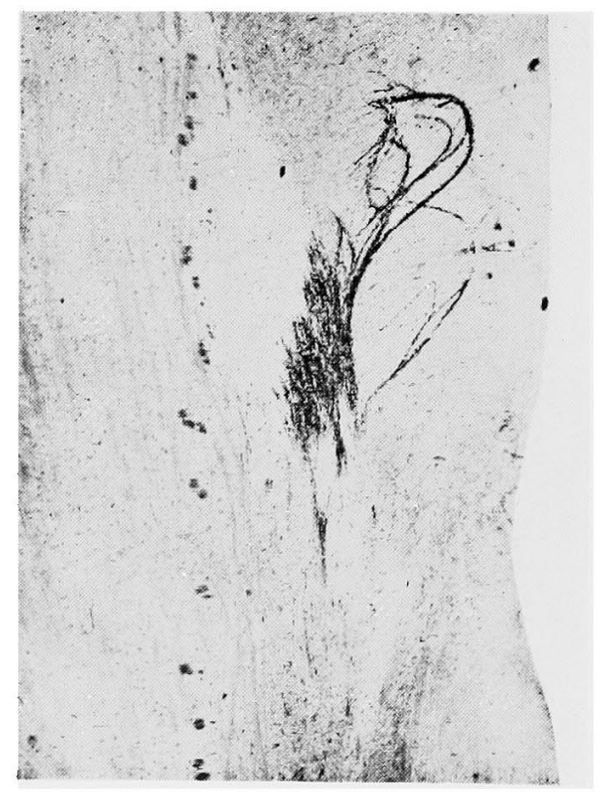

Fig. 4. Dog 22. (90) Magnification of the encircled part in Fig. 3. The fascicles of the degenerated fibres run dorsalward from the rostral part of the solitary tract; some of them make a parabola while others a straight line, terminating in the vestibulospinal tract and in the basal grey substance. Some fibres, after starting from the rostral part of the solitary tract, pass dorsally, make a curve, and enter the lower solitary tract again. 
he fibres starting from the sensory rootlet of the vagus nerve, particularly those rom the rootlet situated on the ventral side, ran dorsocaudally and entered this ateral nucleus (Fig. 5). A small fascicle was seen branching ventrocaudally rom a part of the solitary tract (Fig. 6). There were also scattered black

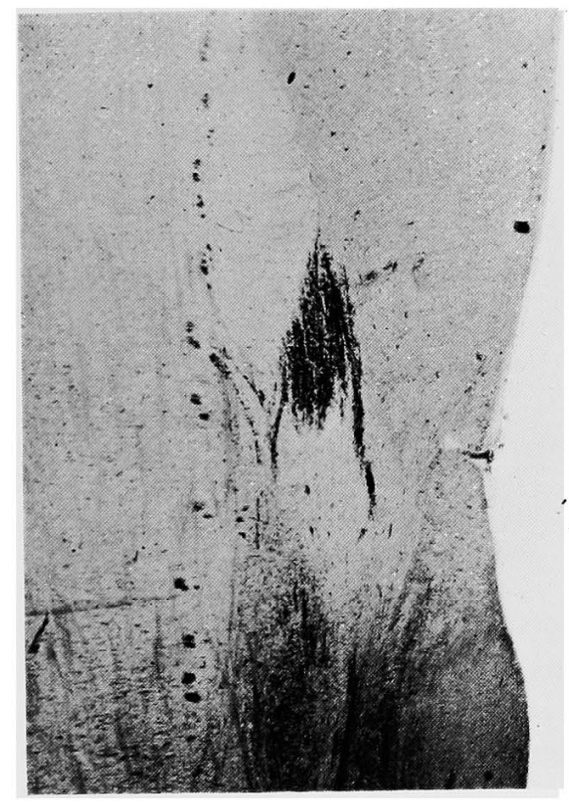

Fig. 5. Dog 22. (93) The big fascicle of the degenerated fibres which appears in the centre of the picture is the solitary tract. The luminous spindleshaped area which is seen on the upper side of the solitary tract is the medial nucleus of the tract. The area which is seen on the lower side of the solitary tract is the lateral nucleus. The large-sized cells are contained, and the fine degenerated granules are conspicuously massed, in this nucleus.

Fig. 6. Dog 22. (99) This section is a more medial one than the section in Fig. 5. A small fascicle of the degenerated fibres runs ventrocaudally from the solitary tract.

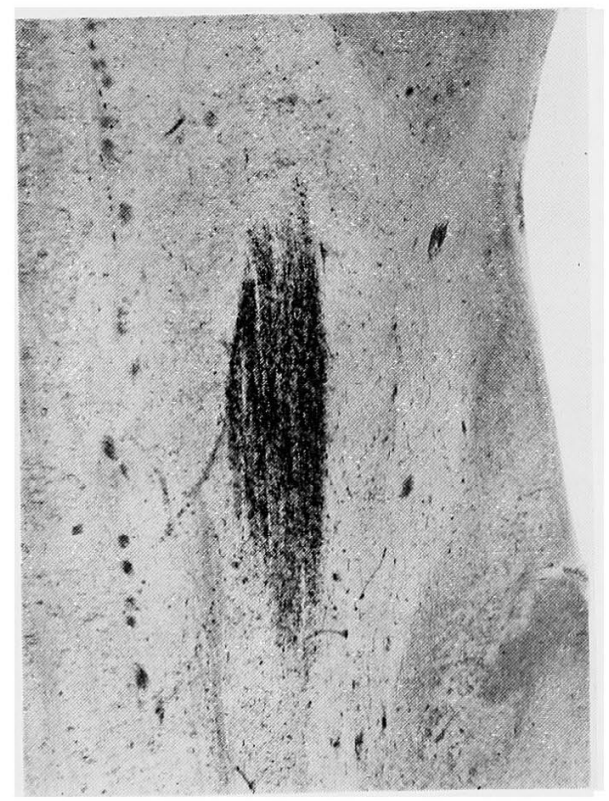


granules in the medial nucleus, in the ventral area of the solitary tract, and in the dorsolateral part of the reticular formation. The dorsal part of the solitary tract came quite near the bottom of the fourth ventricle, and the luminous nucleus of the ala cinerea was seen between the bottom and the tract.

As the section came more medialward, the solitary tract was parallel with the dorsal border of the medulla. The fascicle of the degenerated fibres in the solitary tract ran toward the lower part of the medulla and disappeared at the level of the obex. A little below, the solitary tract disappeared and the degenerated granules also became indistinct except for a small group of fine granules in the commissural nucleus of Cajal.

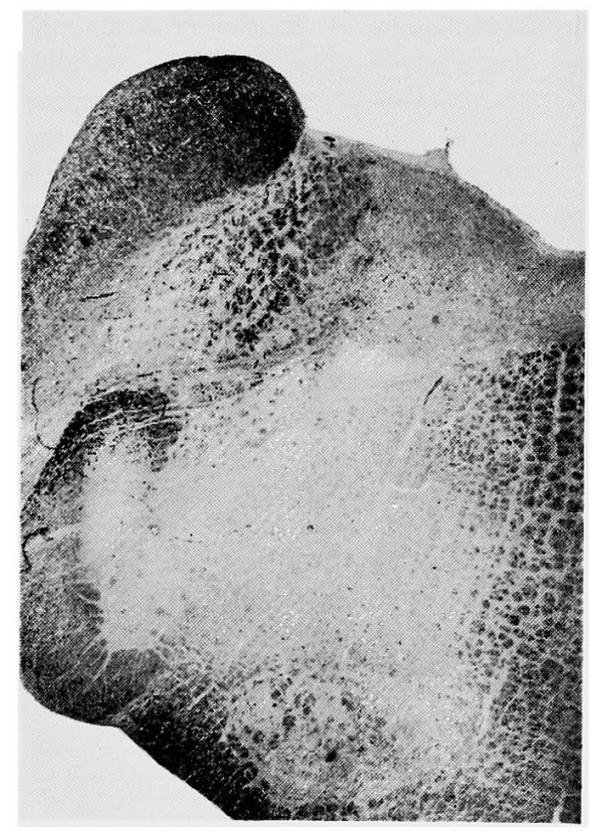

Fig. 7. Dog 18. (88)** This section is the one at the level of the rostral edge of the solitary tract. The newly-discovered course among the vagal afferent components runs dorsalward from the solitary tract, and is distributed clearly to the area where the primary fibres of the vestibular nerve terminate.

**The number in parentheses shows a cross-section of the medulla. Section numbers start from the top of the medulla and end in its caudal edge in the cases of Dogs 18, 19, and 21.

Dog 19. The left vagus nerve was cut between the jugular and nodose ganglia, in addition to the posterior roots of the lumbar spinal cord $\left(L_{4,5,6}\right)$.

The specimens of this case, consisting of the medulla and the spinal cord, were observed in cross-section.

Degeneration of the vagus nerve in the medulla:

Since the observations in cross-section of the medulla on the degenerated fibres after cutting the vagus nerve were previously reported in detail, the authors are going to describe only the gist of the observations in this case. On the sections of the medulla between the level a little below the genu of the facial nerve and the level of the rostral part of the hypoglossal nerve nucleus, degenerated thick fibres in several fascicles ran dorsomedially, rounded the dorsal part of the trigeminospinal tract, and entered the solitary tract from its ventrolateral side 


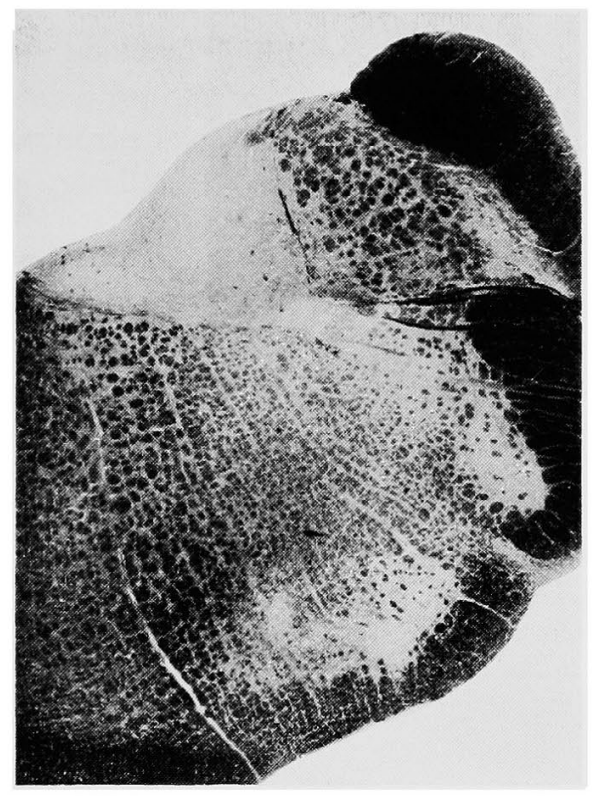

Fig. 8. Dog 19. (16) This section is the one at the level of the most rostral edge of the left solitary tract. A group of the degenerated fibres runs dorsalward through the solitary tract.

(Fig. 8). Most of them turned downward within the tract, giving fine degenerated fibres to the lateral and medial nuclei of the solitary tract. They gradually decreased in number and became smaller in caliber, and a few of them decussated at the commissural nucleus of Cajal (Fig. 11). They completely disappeared at the level of the decussatio pyramidum. At the level where the vagus nerve root entered the medulla, in addition to the degenerated fibres described above, some distinct fascicles of medium-sized or thick degenerated fibres were observed to pass through the solitary tract dorsalward and run toward the lateral border of the basal grey substance along the medial border of the vestibulospinal tract (Fig. 8 ). The whole length of these degenerated fibres was clearly seen in certain sections at the level where the rostral rootlet of the vagus nerve entered the medulla. Some of them ran extremely dorsorostrally and they were traced up to the dorsal nucleus of the acoustic (cochlear) nerve along the dorsal border of the vestibulospinal tract. As the section came down nearer to the hypoglossal nucleus, they became dotted in a line along the lateral border of the basal grey substance until they were barely visible near the solitary tract and finally disappeared. At the level where the caudal sensory rootlet of the vagus nerve entered the medulla, there were a few degenerated fibres which connected the lateral side of the solitary tract with the vestibulospinal tract (Fig. 9). Accessory tracts appeared through nearly the whole length of the solitary tract. But in the rostral part they appeared on the ventral side of the solitary tract; in the caudal part they appeared on the ventrolateral side. They were scattered in a line and touched the dorsal part of the reticular formation (Fig. 10). 


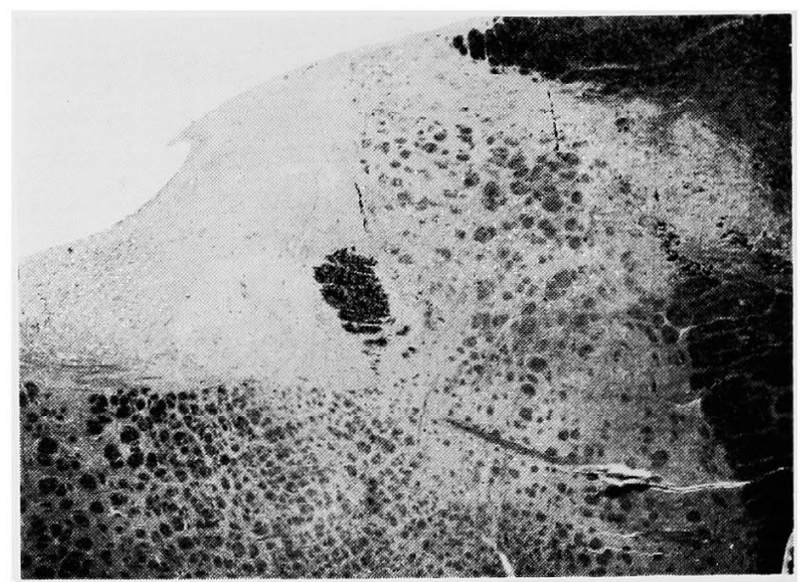

Fig. 9. Dog 19. (58). This is a section at the level where the caudal sensory root of the left vagus enters the medulla. There is a mass of the fine degenerated granules in the solitary tract and there are degenerated fibres running along the lateral border of the vestibulospinal tract.

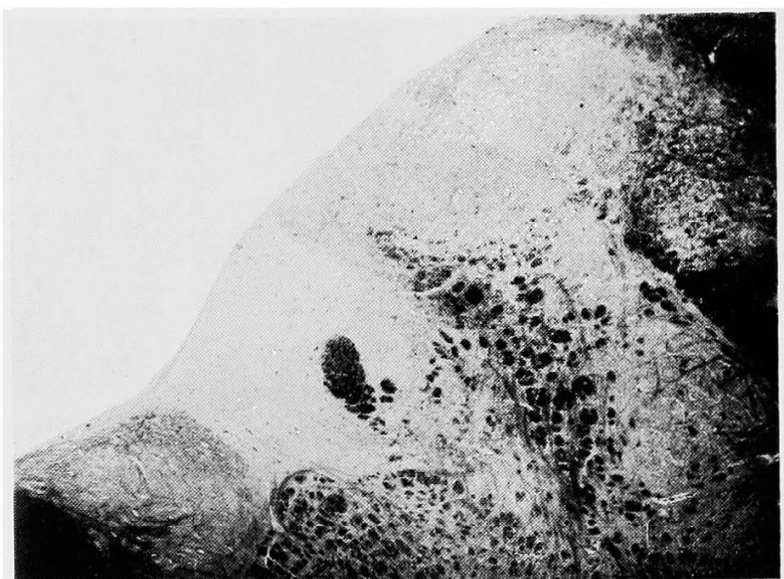

Fig. 10. Dog 19. (110) This is a section at the level where the lateral nucleus of the left solitary tract develops most prominently. There is a luminous nucleus on the side lateral to the solitary tract. There is also a nucleus on the side ventrolateral to the solitary tract. Large-sized and star-shaped cells are contained, and fine degenerated granules are massed, in this nucleus.

Degeneration of the afferent fibres through the spinal cord:

The posterior roots of the left side $\left(\mathrm{L}_{4,5,6}\right)$ of the spinal cord were ascertained to have been cut off. Because strong pressure was applied to the spinal cord for hemostasis during the operation, accidental injuries were seen at the lower part of the lumbar cord. At the level of the lumbar segment, degenerated granules 
were crowded in the bilateral posterior funiculi in cross-section. They were also scattered a little in both the anterior funiculi and the lateral funiculi. Passing rostralward, degenerated afferent fibres became visible in the dorsomedial part of the bilateral posterior funiculi. Degeneration of the afferent fibres through the posterior funiculus was almost the same as in the case of No. 13. The

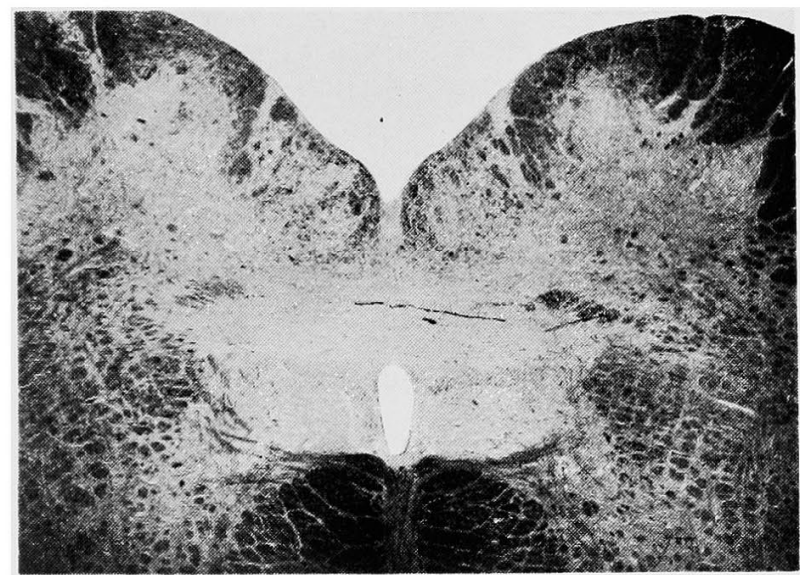

Fig. 11. Dog 19. (171) This is a section at the level a little caudally from the obex. The solitary tract becomes flat at the dorsolateral area of the nucleus alae cinereae. The degenerated granules are still seen around the solitary tract on the left side, some of them passing to the solitary tract on the right side through the commissural nuclei of Cajal. In Goll's nucleus (nucleus fasciculi gracilis), there are scattered degenerated granules caused by severing the lower spinal cord.

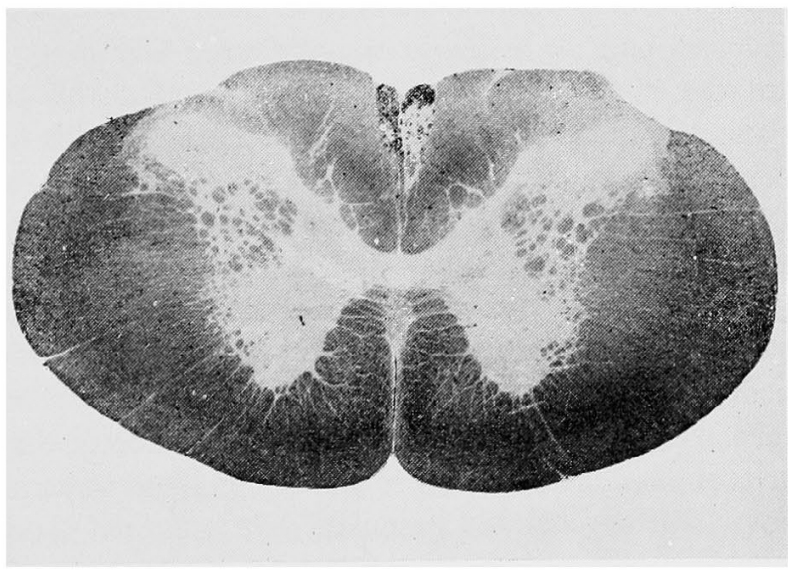

Fig. 12. Dog 19. This is a cross-section at the level of $C_{1}$. The degenerated granules are massed in both of Goll's fasciculi (fasciculus gracilis). A little degeneration is seen in both lateral funiculi. 


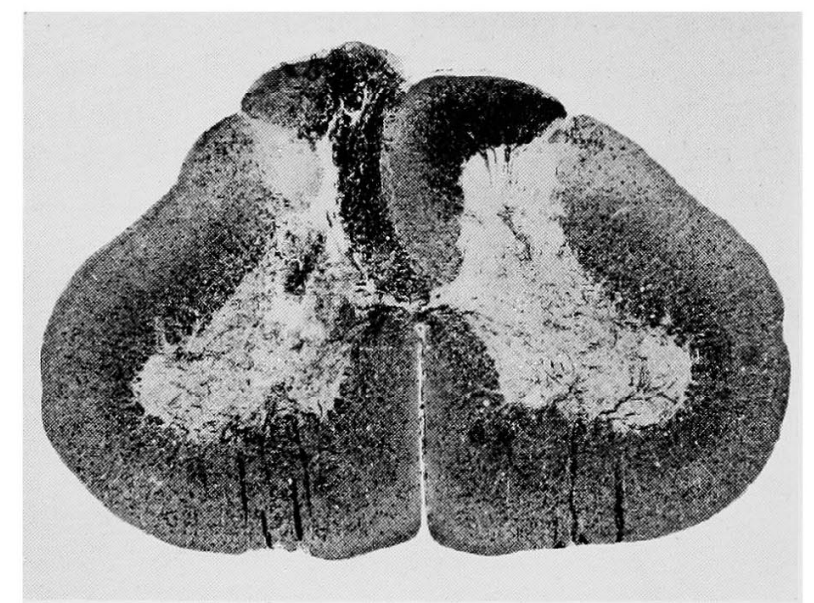

Fig. 13. Dog 19. This is a cross-section at the level of the lumbar cord $\left(L_{6}\right)$ where the damage was induced. Remarkable degeneration is seen in the left dorsal funiculus (mostly around the entrance of the posterior root) and in the right dorsal funiculus (mostly the medial area). There is also a little degeneration in the lateral funiculus and in the anterior funiculus.

degenerated granules which were scattered in the lateral and anterior funiculi, decreased in number as the segment went upward (Fig. 11, 12, 13).

\section{Dog 17. The left cochlea was damaged.}

The specimen, that is, the medulla of this case, was observed in cross-section.

At the level where the left acoustic nerve entered the medulla, medium-sized or fine degenerated granules were gathered in the ventral nucleus of the cochlear nerve, and thick degenerated fibers were seen to pass dorsomedially between the cochlear nerve nucleus and the trigeminospinal tract. The most lateral of these fibres ran toward the cerebellum via the restiform body, while the medial one ran toward the lateral bottom of the fourth ventricle. From this level caudalward the thick degenerated fibres, after going round the dorsal side of the restiform body, entered the vestibulospinal tract and its nucleus where they terminated in intricately formed large- or medium-sized granules. The fine degenerated granules were gathered in the area of the triangular nucleus on the lateral side of the basal grey substance. At the level of the rostral edge of the solitary tract, a number of medium-sized or fine degenerated fibres ran dorsomedially toward the basal grey substance, starting from the ventromedial side of the vestibulospinal tract and its nucleus. Just below these sections, the same observation was made as described above, and degenerated granules were scattered around the solitary tract, also. As the section went lower, the vestibulospinal tract and its nucleus became smaller in size, and the degenerated granules in these areas decreased in number. At the level of the edge of the inferior olivary nucleus, there was nothing 
but a small group of scattered fine granules in the dorsal area of the solitary tract and in the vestibulospinal tract. Below the obex, no degenerated granule was recognizable.

\section{Dog 18. The right cochlea was destroyed.}

The specimen of the case was observed in cross-section.

Degeneration in this case was almost the same as in the case of No. 17, except that a group of the degenerated fibres was observed to pass through a quarter of the dorsal part of the right trigeminospinal tract down to the upper cervical spinal cord (Fig. 7).

Dog 13. The posterior roots of the right side of the sacral spinal cord were severed.

In this case, the specimens, that is, the medulla and the spinal cord, were observed in cross-section.

At the level of $L_{1}$, a cut was made in the shape of a wedge from the entrance of the posterior root toward the ventral part, the damage extending to the entire area of the lateral funiculus, posterior column, the intermediate zone, and even to a part of the anterior nucleus. The degenerated afferent fibres were observed mostly in the circumference of the right lateral funiculus. (A few of the degenerated granules, seen in the dorsomedial part of the posterior funiculus, were due to the damage of the posterior root of $L_{1}$.) Most of the medium-sized or thick degenerated fibres ran in groups up through the most dorsal part of the lateral funiculus, near the entrance of the posterior root. At the level of the upper cervical cord, fine degenerated granules appeared in addition to the medium-sized or large granules. These fine granules were scattered, even in the lateral corticospinal tract (pyramidal tract) and in the dorsolateral part of the reticular formation. At the level of the medulla, the thick degenerated fibres gradually moved dorsalward, ascended through the restiform body, and most of them ran toward the cerebellum. The pathway of the degenerated fibres through the restiform body was interesting to note. Some of them moved dorsomedially, left the restiform body at the level of the obex, and ran through the dorsal border of the medulla toward the triangular area which was adjacent to the ala cinerea on the dorsal side of the solitary tract. Passing rostralward, medium-sized or fine degenerated granules were massed, forming a band-like shape in the area between the dorsomedial border of the restiform body and the nucleus of the ala cinerea. Further rostralward, the granules began to decrease in the part adjacent to the ala cinerea, and finally disappeared at the level where the nucleus of the ala cinerea became smaller.

A group of the degenerated fibres which ascended the anterior part of the lateral funiculus of the spinal cord became adjacent to the nucleus of the lateral funiculus and the inferior olivary nucleus in the medulla, and further ran rostralward through the lateral funiculus, giving off fine granules to the reticular formation. A few degenerated fibres which originated in the accidentally injured posterior 
root of $\mathrm{L}_{1}$ ascended through the posterior funiculus, showing almost the same pathway as in the case of No. 13, until they joined the degenerated fibres from the lateral funiculus and the restiform body at the level of the rostral side of the obex.

Dog 20. The left vagus nerve was cut between the jugular and nodose ganglia, in addition to the left posterior roots of the thoracic spinal cord.

The specimens of this case, that is, the medulla and the spinal cord, were studied in cross-section.

Degeneration of the vagus nerve in the medulla:

Degeneration caused by cutting the vagus nerve between the ganglia was almost the same as in the case of No. 19.

Degeneration of the afferent fibres through the spinal cord:

The left posterior roots of the thoracic spinal cord $\left(\mathrm{Th}_{2,3}, 4,5\right)$ were ascertained have been completely severed. In the sections at a level more rostral than $T h_{2,3,4}, 5$, the degenerated fibres moved medialward through the posterior funiculus, and showed a slender wedgelike shape in cross-section, and came near to the lateral border of the fasciculus gracilis. When the sections were made at a level above the 4 th cervical cord, the granules of the degenerated fibres which ascended the border of the posterior funiculus had a tendency to move ventrolateralward until they lay scattered as far as the entrance of the posterior root. Large or fine degenerated granules were massed in the shape of a band in crosssection along the medial- and dorsal-borders of the cuneiform fasciculus. In the medulla, the degenerated granules in the medial border of the cuneiform fasciculus became much finer and entered the nucleus of the cuneiform fasciculus. The degenerated fibres in the dorsolateral border of the cuneiform fasciculus ascended without any change of number and size. However, at the level above the obex they became smaller and entered the nucleus of the cuneiform fasciculus.

Further rostralward, the nucleus of cuneiform fasciculus became so small as to be enclosed by the restiform body, the vestibulospinal tract and its nucleus. The degenerated granules in it decreased in number, and at the level of the hypoglossal nerve nucleus they were scarcely visible in the area encircled by the dorsal side of vestibulospinal tract and dorsomedial border of the restiform body.

Dog 21. The left vagus nerve was cut between the ganglia, in addition to a quarter of the right anterior area of the thoracic spinal cord and the right posterior roots of the thoracic spinal cord.

The specimens of this case, that is, the medulla and the spinal cord, were examined in cross-section.

Degeneration of the vagus nerve in the medulla:

Degeneration caused by cutting the vagus nerve between the ganglia was almost the same as in the cases of Nos. 19 and 20 .

Degeneration of the afferent fibres through the spinal cord: 
It was ascertained that, at the level of $\mathrm{Th}_{6}$, a quarter of the right anterior area of the spinal cord had been clearly severed. The cut extended from the directly dorsal part of the right ligamentum denticulatum to a part of the left anterior funiculus. The posterior root of the right $\mathrm{Th}_{6}$ had also been severed (Fig. 18). The same findings as in the case of No. 20 were observed concerning the degenerated fibres which originated in the posterior root, and ascended through the posterior funiculus. It was interesting to examine the afferent degenerated fibres through the lateral and the anterior funiculi. Although the cut was made to the anterior half of the spinal cord at the level of $\mathrm{Th}_{6}$, the degenerated granules were massed mostly in the lateral border of the spinal cord, and the large-sized granules were observed, also, in the lateral border of the posterolateral funiculus, as the cross-section moved upward. These granules moved near to the ventral side of the posterior horn at the upper cervical cord, and the further course of the fibres was traced almost in the same way as in the case of No. 15. However, since no degeneration was seen in the fasciculus gracilis in this case, the course and termination of the fibres through the lateral funiculus was clearly seen in the medulla. A number of the thick degenerated fibres, ascending through the posterolateral funiculus, ran toward the cerebellum via the restiform body; a part of them, passing through the restiform body, ran along the lateral border of the medulla and terminated in the triangular area which was adjacent to the dorsolateral side of the ala cinerea. This triangular area began from the fasciculus gracilis and its nucleus at a level a little below the obex, and came near the dorsal side of the solitary tract and the dorsolateral corner of the ala cinerea. As the level of the section came upward, it moved lateralward and developed

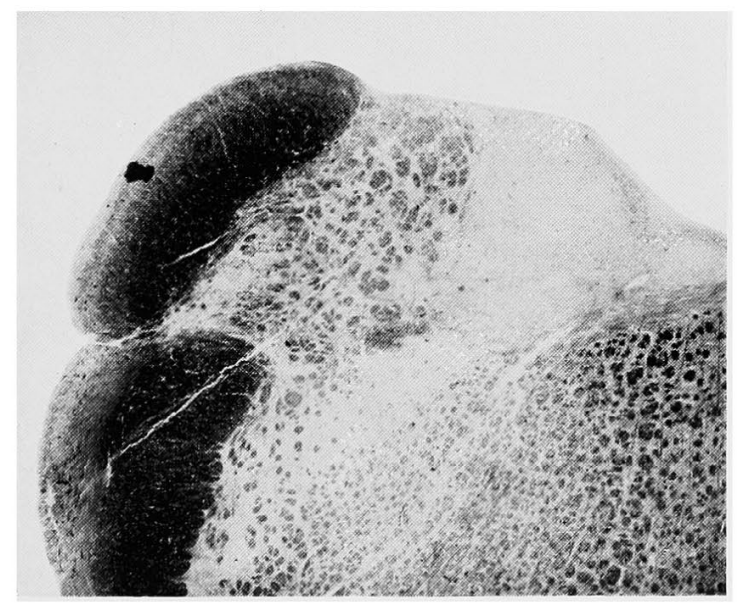

Fig. 14. Dog 21. (14) This section is the one at the level of the most rostral edge of the right solitary tract. No degeneration is seen in the solitary tract, in the basal grey substance, or in the vestibulospinal tract. 
in proportion to the development of the ala cinerea. It was pushed dorsalward by the appearance of the triangular nucleus, and gradually decreased in size. There were no degenerated granules in it, but some were seen in the restiform body at the level of the rostral edge of the hypoglossal nerve nucleus (Fig. 14, $15,16,17)$.

A few thick degenerated fibres were observed in the dorsolateral area of the solitary tract at the level a little below the obex; they ran dorsomedialward along the ala cinerea and entered the nucleus of the fasciculus gracilis. Some of them passed through the dorsal side of the commissural nucleus of Cajal and a

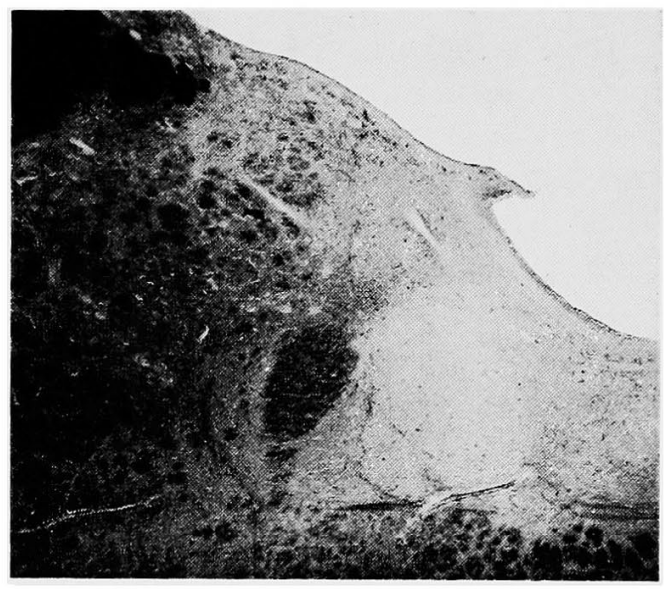

Fig. 15. Dog 21. (50) This is a section at the level where the sensory caudal root of the right vagus nerve enters the medulla. The degenerated granules are scattered over the area where the restiform body, the dorsal part of the vestibulospinal tract, and the chorioidal tissue of the fourth ventricle are involved.

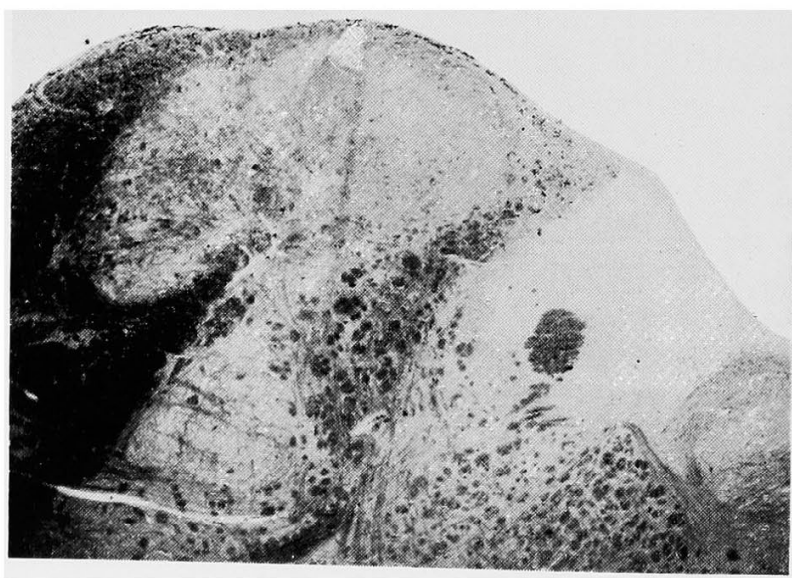

Fig. 16. Dog 21. (81) This is a section at the level where the lateral nucleus of the solitary tract develops most prominently. Many large or fine degenerated granules are seen in the triangular area, which is adjacent to the dorsolateral side of ala cinerea. 


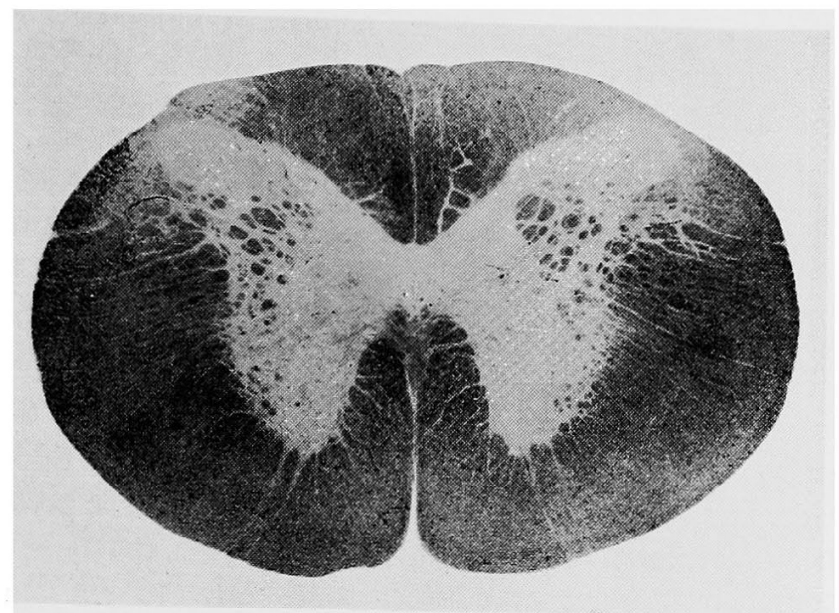

Fig. 17. Dog 21. This is a cross-section at the level of $C_{1}$. The degenerated granules are seen in the posterolateral funiculus and in the anterolateral funiculus, of the right side

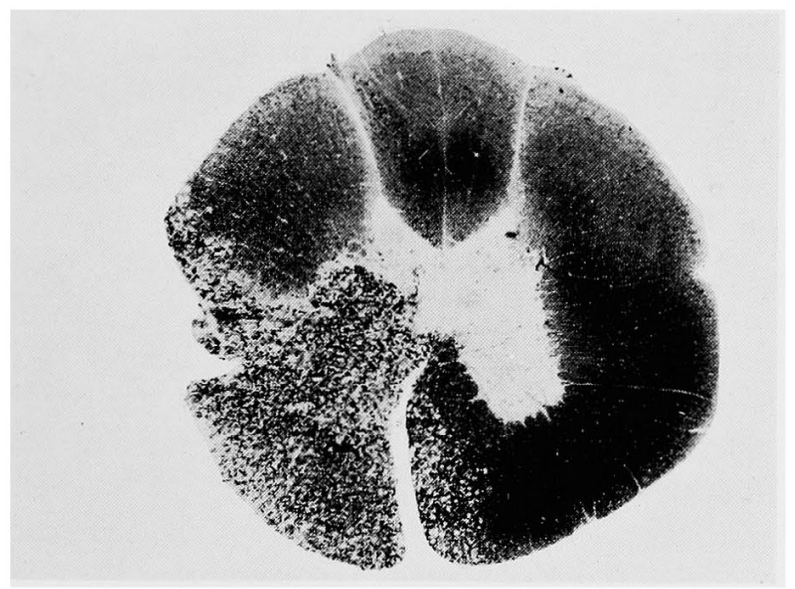

Fig. 18. Dog 21. This is a cross-section at the level of the thoracic segment of the spinal cord $\left(\mathrm{Th}_{6}\right)$, where the damage was done. A quarter of the right anterior area of the spinal cord is damaged and a part of the left anterior funiculus is also damaged. The posterior root of the right $\mathrm{Th}_{6}$ is also severed.

few seemed to decussate. A little caudally from the level where the most caudal sensory rootlets of the vagus nerve entered the medulla, a few thick degenerated fibres were observed to pass between the trigeminospinal tract and the nucleus of the posterior funiculus. There were also some fibres which passed through the nucleus of the posterior funiculus and ran ventromedially toward the lateral side of the solitary tract. At the level where the most caudal sensory rootlet entered 
the medulla, the medium-sized or fine degenerated granules were adjacent to the dorsomedial side of the restiform body. The pathway of the degenerated fibres which ascended through the anterior part of the lateral funiculus was almost the same as in the case of No. 15.

\section{Discussion}

It has generally been held that the vagus nerve plays an important role in respiration-, circulation-, and digestion-reflexes. It has also been known that visceral afferent components of the vagus nerve have much to do with respiration, coughing, sneezing, swallowing, nausea, vomiting, hunger- and thirst-feelings, and that they have contact of some kind with atrial receptors, aortic pressure receptors, and aortic chemoreceptors. Despite such complicated functions of the vagus nerve, all authors have so far been satisfied with the simple conclusion about the pathway of this nerve; that visceral afferent components of the vagus nerve enter the solitary tract and terminate in the solitary nuclei.

Now, two problems can be raised:

(1) It is too simple to conclude that these important afferent fibres terminate in the medial and the lateral nuclei of the solitary tract only.

(2) The reflexes which are related to the vagus nerve are so complicated that they are regarded as a chain reflex. Therefore, the afferent fibres of the vagus nerve must have a close relation not only to the solitary nuclei, but also to the other nuclei around the solitary tract.

As for the first problem, only two nuclei in the rostral part of the solitary tract have hitherto been noticed. They are the superior salivatory nucleus and Grossman's nucleus for gustatory sensation, which belong to the intermediate nerve and the glossopharyngeal nerve. However, there has been no report about the functional difference according to the different parts of the solitary nuclei which appear through nearly the whole length of the medulla. The present authors, in the previous report, described the degenerated granules in the solitary nuclei caused by cutting the vagus nerve, and stated that there was a remarkable difference of morphology according to the different levels not only of the medial nucleus but also of the lateral nucleus. It was suggested that there should be functional differences among the parts of the nuclei where morphological differences were observed. Further studies will be necessary on this subject from anatomical and physiological aspects. As for the second problem, it was reported by some authors that a part of the sensory components of the vagus nerve entered the following nuclei:- the sensory nuclei, such as nucleus intercalatus (DuBois, 1929; Barnard, 1940; Moffle, 1942; Kappers, 1947), and nucleus praepositus hypoglossi (Barnard, 1940; Moffle, 1942; Kappers, 1947); and the motor nuclei such as the dorsal vagal nucleus (Allen, 1923), and the nucleus ambiguus (Mettler, 1942). Their reports, however, were not generally accepted. The present 
authors, in the previous report, described in detail that the somatic sensory pathway among the degenerated fibres (which were caused by cutting the vagus nerve and originated in the jugular ganglion) passed through the trigeminospinal tract.

From the degeneration experiments on dogs, the present authors have discovered the distinct course of the fibres in the sensory afferent components of the vagus nerve, which had not been noticed before by any authors. A distinct group of thick degenerated fibres, caused by cutting the vagus nerve, was recognized to pass the dorsomedial part of the solitary tract, and run dorsalward along the lateral border of the triangular nucleus at the level where the rostral rootlet of the vagus nerve entered the medulla. Some of the fibres ran downward making a parabola curve, and at the level where the caudal rootlet of the vagus nerve entered the medulla, joined the solitary tract again, giving off the degenerated fibres into the triangular nucleus and the vestibulospinal tract. Some of the fibres ran extremely dorsalward and were traced to the area of the dorsal nucleus of the cochlear nerve. It was ascertained from the observations of the sagittal sections that the degenerated fibres, which started from the rostral part of the solitary tract, went down with a parabola curve and joined the tract again, had contact with the large-sized cells in the lateral nucleus of the solitary tract as well as with the dorsolateral part of the reticular formation which is adjacent to the ventrolateral side of the solitary tract (Fig. 19).

It was generally considered that the primary fibres of the vestibular nerve terminated in all four vestibular nerve nuclei. However, Leidler (1914) and Lorente de Nó (1933) noticed that an area lacked the degenerated fibres in the vestibular nerve nucleus after the vestibular nerve had been cut. Walberg, Bowsher, and Brodal (1958) pointed out cell-groups $\mathrm{X}$ and $\mathrm{Z}$ in the vestibular nucleus of mice where the degenerated granules were not present after cutting the vestibular nerve. The present authors also recognized that the corresponding area in dog's vestibular nucleus to mice's cell-groups $\mathrm{X}$ and $\mathrm{Z}$ was lacking in the primary fibres of the vestibular nerve. Much interest will be aroused if the lacking area can be proven to have some relations to the afferent fibres from the spinal cord.

Thiele and Horsley (1901) first reported that some of the afferent fibres through the lateral funiculus of the spinal cord terminated in the dorsal part of the medulla. The outstanding authors who thereafter studied this subject were Collier and Buzzard (1903), MacNalty and Horsley (1909), Lorente de Nó (1924), Kuru (1940, 1956), and Pompeiano and Brodal (1957). Most of the authors thought of the communication between the spinal cord and the vestibular nerve as a spinovestibular system. Kuru looked upon this communication, together with the ventral and dorsal collateral plexuses by Thiele and Horsley, as the tract between the spinal cord and the medulla, and he assumed this tract was a visceral, particularly a pelvic visceral, sensory pathway. The nucleus paraalaris named by Kuru (the termination of the spinobulbar tract) nearly 


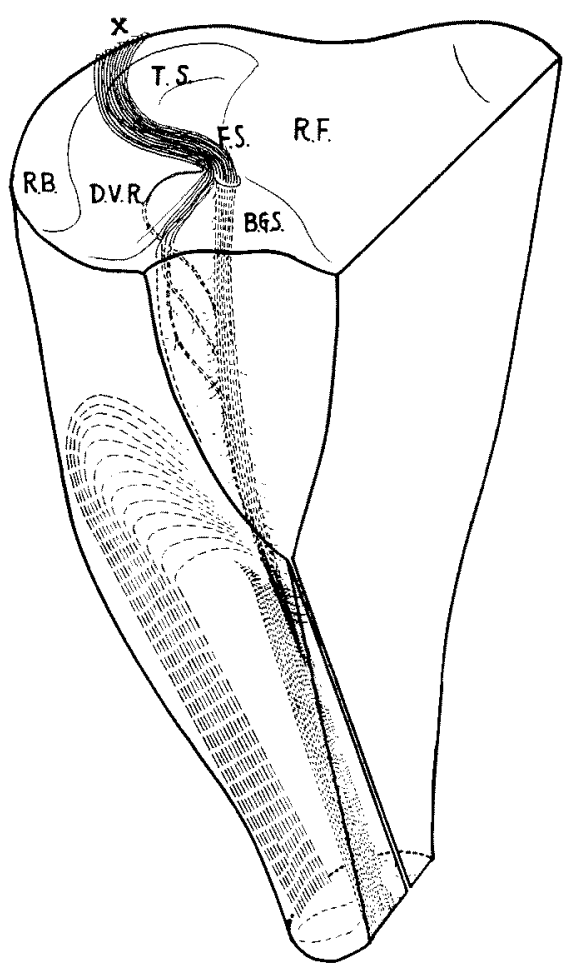

Fig. 19. Schematic illustration showing the afferent course of the vagus nerve and the relationship between the vagal afferent fibres and the ascending fibres from the lower spinal cord.

Sensory course of the vagus nerve Afferent course passing through the lateral funiculus of the spinal cord Afferent course passing through the posterior funiculus of the spinal cord

$X$.: The vagus nerve

R. B.: Restiform body

R. F.: Reticular formation

F.S.: Solitary tract

B. G.S.: Basal grey substance

T.S.: Trigeminospinal tract

D. V.R.: Vestibulospinal tract.

corresponded to cell-group $\mathrm{Z}$ by Pompeiano and Brodal. According to the present authors' experiments, this area in dog also was lacking in the primary gbres of the vestibular nerve. Furthermore, the degenerated fibres through the lateral funiculi of the spinal cord, passing the restiform body, terminated in the area wider than the nucleus paraalaris,-rather in the area corresponding to cell-group $\mathrm{X}$ and $\mathrm{Z}$ by Pompeiano and Brodal.

The newly-discovered course by the present authors was clearly distributed to the vestibulospinal tract and the triangular nucleus: the area where the primary fibres of the vestibular nerve terminated. On the other hand, some of the ascending fibres through the lateral funiculus of the spinal cord terminated in the area near the dorsal side of the solitary tract and the lateral side of the ala cinerea at a level a little rostralward from the obex (Fig. 19). It was very significant that the pathway of these afferent fibres through the lateral funiculus ran parallel with, and was situated on the dorsocaudal side of, the newlydiscovered course.

The newly-discovered course is to be considered as having something to do with the digestion organs, because the course originates in the nodose ganglion which regulates the visceral afferent components, and because the course is also related to the glossopharyngeal nerve which regulates the sensory path of 
the pharynx. Dr. Sugihara, one of the co-workers, while making observations of the central regulator of the stomach and intestine of cat, noticed the vomiting reflex was caused by stimulation of the area with which our new course was supposed to have some relation. Dr. Sugihara's area was supposed to have contact with the most rostral part of the "Nucleus paraalaris" which was closely related to the lower spinal cord. The newly-discovered course was adjacent to cell-group $\mathrm{X}$ and $\mathrm{Z}$, or nucleus paraalaris, and also was closely allied with the vestibular nerve. The newly-discovered course, therefore, is considered as having to do with vomiting which is a combination of reflexes from various routes. Since Dr. Thumas's study (1891), the vomiting centre has been identified with this area by Miller and Sherrington (1916), Hatcher and Weiss (1924), Borison and Brizzee (1951), Borison and Wang (1951), and Borison (1957).

According to our experiments, the newly-discovered course could not be found in rabbit which does not vomit, and it was interesting to notice that this course was observed in dog and cat which do vomit. Spitzer's assertion that the triangular nucleus is the "Vestibulär-alimentäres Reflexzentrum" should be remembered in this connection.

\section{CONCLUSION}

The intramedullary course of the vagus nerve was studied by means of degeneration caused by cutting the vagus nerve.

A group of distinct thick degenerated fibres was newly discovered which ran dorsalward from the solitary tract. These degenerated fibres passed the solitary tract at its rostral edge and ran dorsalward along the lateral border of the basal grey substance. Some of them from there went downward, making a parabola curve, and giving off fine degenerated fibres to the triangular nucleus and the other nucleus of the vestibulospinal tract. Some of them joined the solitary tract again at the level where the rostral rootlet of the vagus nerve entered the medulla. The newly-discovered course was confined within the area where the degenerated granules were visible after cutting the vestibular nerve. The newly-discovered course did not have a direct contact with the ascending fibres from the lower spinal cord. However, the afferent fibres through the spinal cord ran parallel with the new course and terminated quite near the new course on its dorsocaudal side.

\section{REFERENCES}

1) AlLes, W.F.: Origin and distribution of the tractus solitarius in the guinea pig. J. Comp. Neur., 35, 171-204, 1923.

2) BARNARD, J.W.: A phylogenetic study of the visceral afferent areas associated with the facial, glossopharyngeal and vagus nerves and their fibre connections. Ibid., 65, 503-602, 
1936.

3) BoRIsON, H. L.: Site of emetic action of X-radiation in the cat. Ibid., 107, 439-453, 1957.

4) BoRISON, H.L. and K.R. BRIZZEE: Morphology of emetic chemoreceptor trigger zone in cat medulla oblongata. Proc. Soc. Exper. Biol. Med., 77, 38-42, 1951.

5) Borison, H.L. and S. C. WANG: Locus of the central emetic action of cardiac glycosides. Ibid., 76, 335-338, 1951.

6) Collier, J. and E.F. Buzzard: The degeneration resulting from lesions of posterior nerve roots and from transverse lesions of the spinal cord in man. A study of twenty cases. Brain, 26, 559-591, 1903.

7) DuBors, F.S.: The tractus solitarius and attendant nuclei in the virginian opposum (Didelphis virginiania). J. Comp. Neur., 47, 189-224, 1929.

8) Hatcher, R. A. and S. Weiss: Studies on vomiting. J. Pharmacol, 22, 139-193, 1924.

9) Kappers, C. U. Ariens: Anatomie comparee du systeme nerveux. Masson et Cie, Paris, 1947.

10) KURU, M.: Ueber die bulbären Endigungen des anterolateral ascendierenden Bündels, unter besonderer Berücksichtigung eines neuen spino-bulbären Systems-des Tractus spinojuxtasolitarialis. Jap. J. Med. Sc., Part I, Anat., 8, 135-160, 1940.

11) KuRU, M.: The spino-bulbar tracts and pelvic sensory vagus. Further contributions to the sensory dual innervation of the viscera. J. Comp. Neur., 104, 207-232, 1956.

12) LeIDLER, R.: Experimentelle Untersuchungen über des Endigungsgebiet des Nervus vestibularis. Arb. Neurol. Inst., Wien, 21, 151-212, 1914.

13) LoREnTE de Nó, R.: Etudes sur le cerveau posterieur. Trav. Lab. Rech. Biol., Madrid, 22, 51-65, 1924.

14) LORENTE DE Nó, R.: Anatomy of the eighth nerve. The central projection of the nerve endings of the internal ear. Laryngoscope, 43, 1-38, 1933.

15) MACNALTY, A.S. and V. Horsley: On the cervical spino-bulbar and spino-cerebellar tracts and on the question of topographical representation in the cerebellum. Brain, 32, 237-255, 1909.

16) Metrler, F. A.: Anatomy of the nervous system. C. V. Mosby Co., St. Louis, 1942.

17) Miller, F.R. and C.S. Sherrington: Some observations on the buccopharyngeal stage of reflex deglutition in the cat. Quart. J. Exper. Physiol., 9, 147-186, 1916.

18) MoFfle, D.: The comparative anatomy of the nucleus intercalatus (Staderini) and adjacent structures. Van Gorcum and Comp. N. V., Assen., 1942.

19) Pompeiano, O. and A. Brodal: Spino-vestibular fibres in the cat. An experimental study. J. Comp. Neur., 108, 353-382, 1957.

20) SAWADA, K.: On comparative anatomy of nucleus paraalaris (In Japanese). Juzenigakukai Zasshi, 55, 869-891, 1953.

21) Sprtzer, A.: Anatomie und Physiologie der zentralen Bahnen des Vestibularis. Arb. Neurol. Inst., Wien, 25, 422-470, 1924.

22) SugIHARA, S.: Responses of the stomach and intestine caused by electrical stimulation of the medulla (In Japanese). Juzen-igakukai Zasshi, 56, 329-338, 1954.

23) Thiele, F.H. and V. Horsley: A study of the degenerations observed in the central nervous system in a case of fracture dislocation of the spine. Brain, 24, 519-531, 1901.

24) URABE, M., S. YAMAZAKI and K. ARAKI: The central course of afferent fibres of the glossopharyngeal and vagus nerves. Neurologia medicochirurgica, 2, 1-24, 1960.

25) Walberg, F., D. Bowsher and A. Brodal: The termination of primary vestibular fibres in the vestibular nuclei in the cat. An experimental study with silver methods. J. Comp. Neur., 109, 391-416, 1958. 